\title{
Fermi-LAT constraints on diffuse Dark Matter annihilation from the Galactic Halo
}

\section{Brandon ANDERSON*}

University of California - Santa Cruz

E-mail: anderson@physics.ucsc.edu

Our Galaxy resides in the center of a vast "Halo" of Dark Matter (DM). This concentration produces, in many viable particle physics models, an indirect Weakly Interacting Massive Particle (WIMP) annihilation signal that peaks in the Fermi-LAT's energy range. Our knowledge of the diffuse background is essential to placing reasonable limits on the DM mass and cross-section. We incorporate a systematic variation of the GALPROP galactic diffuse background model, constrained by current cosmic-ray measurements, into a profile likelihood analysis and present preliminary upper limits on the DM annihilation cross-section using the Fermi-LAT data.

Identification of Dark Matter 2010-IDM2010

July 26-30, 2010

Montpellier France

*Speaker.

${ }^{\dagger}$ On behalf of the Fermi-LAT Collaboration. 


\section{Signal}

The Milky Way rests at the center of a massive, $\mathscr{O}\left(10^{12}\right) M_{\odot}$ [Diemand et al. (2007)], Halo of Dark Matter (DM). We have a good sense of the large-scale mass distribution of this Halo from $\mathrm{N}$-body simulations, and can approximate that with a simple density profile function. For example, here we use an Einasto profile, where $\rho(r) \propto \exp \left(-A r^{\alpha}\right)$. If this Halo is comprised of Weakly Interacting Massive Particles (WIMPs) capable of annihilation or decay into Standard Model (SM) particles, the Fermi-LAT provides a unique all-sky energy window to search for gamma-ray signal resulting from that process.

To capture the uncertainty in our DM signal, we consider WIMPs of mass 25 to $500 \mathrm{GeV}$ annihilating to a variety of SM channels, including $b \bar{b}, t \bar{t}, \tau^{+} \tau^{-}$, and $\mu^{+} \mu^{-}$. And while N-body simulations are powerful, their inability to resolve small scale structure leaves another uncertainty in the "boost" that comes from extrapolating their mass functions down to the free-streaming cutoff. We therefore use three different mass function exponents $(1.8,1.9,2.0)$ to cover the reasonable [Diemand et al. (2008)] range of possibilities.

While channels like $b \bar{b}$ deliver their gamma-ray signal mostly through $\pi^{0}$ decay, others (e.g. $\mu^{+} \mu^{-}$) require an extra step in calculating the predicted Fermi-LAT signal. This is the propagation and interaction of the final SM decay products. This calculation is performed with the same methodology as the rest of our cosmic-ray (CR) backgrounds discussed in the next section.

\section{Diffuse Backgrounds}

Since we expect the Halo signal to be large-scale and slowly varying, masking out regions of the sky containing point sources greatly simplifies our analysis without much affecting our sample size. We mask the entire galactic plane to $\pm 10^{\circ}$, and Fermi sources with an energy-dependent sized mask corresponding to the LAT PSF.

After masking, we contend only with diffuse backgrounds to the Halo signal: extragalactic, instrumental, point source residuals, and galactic diffuse. The first two enter the fit as completely isotropic signals with fixed energy spectra [Abdo et al. (2010)]. Point source residuals, products of the extended tails of the PSF that reach outside our masking scheme, are modeled using FermiLAT software ${ }^{1}$ tuned to the Fermi First Source Catalogue [Abdo et al. (2010)] measured source parameters.

Originating from interactions (bremsstraulung, inverse compton, and $\pi_{0}$ decay) of CR with the gas, light, dust, and magnetic fields in the Milky Way, the galactic diffuse is by far the most difficult background to model. GALPROP [Strong et al. (2000)] is a code designed to self-consistently solve the transportation and interaction of $\mathrm{CR}$ within the galaxy from source injection to arrival at Earth radius. We assess the quality of a particular realization of GALPROP's parameter space by comparing with available CR measurements. This is advantageous in the respect that it makes our model independent of the gamma rays we wish to probe for DM signals.

The large number and uncertainty of parameters in the GALPROP framework, however, requires us to make a thorough investigation of that space to quantify our systematics. To that end,

\footnotetext{
${ }^{1}$ http://Fermi.gsfc.nasa.gov/ssc
} 


\begin{tabular}{|l|l|}
\hline Parameter & Range \\
\hline \hline Diffusion Coefficient & $1 \times 10^{27} \rightarrow 4 \times 10^{29}$ \\
Halo Height & $1 \rightarrow 11 \mathrm{kpc}$ \\
Diffusion Index & $0.33,0.50$ \\
Alfven Velocity & $0 \rightarrow 50 \mathrm{~km} \mathrm{~s}^{-1}$ \\
Electron Injection Index & $1.8 \rightarrow 2.5$ \\
Nucleon Injection Index (Low) & $1.7 \rightarrow 2.6$ \\
Nucleon Injection Index (High) & $2.26,2.43$ \\
Source Distribution & Parameterized, SNR, Pulsars \\
\hline
\end{tabular}

Table 1: GALPROP parameter space.

we fit with models generated within the parameter space in Table 1. Each model's validity is quantified by a $\chi^{2}$ fit to ${ }^{10} \mathrm{Be} /{ }^{9} \mathrm{Be}, \mathrm{B} / \mathrm{C}$, and proton data from the HEAO-3, IMP, ATIC-2, CREAM, ACE, ISOMAX, AMS01, CAPRICE, and BESS experiments. The explicit form is,

$$
\chi^{2}=\Sigma_{j} \Sigma_{i}^{N j} \frac{\left(D_{i j}-T_{i j}\right)^{2}}{\sigma_{i j}^{2}+\Delta \phi_{i j}^{2}},
$$

where $\mathrm{D}$ and $\mathrm{T}$ are model and data respectively, $\Delta \phi^{2}$ comes from uncertainty in the solar modulation (taken to be $\pm 100 \mathrm{MV}$ ), and the sum is over all points and experiments.

One uncertainty obviously missing from the parameter scan is the normalization of the primary electron sources. The sensitivity of a local measurement to nearby sources (or lack of) makes this a particularly difficult value to set a parameter range for. Running each model with and without primary electrons and finding the difference isolates their contribution so that we can let it float freely in our fits to the data.

\section{Profile Likelihood}

After binning into 12 annular and 80 logarithmic energy bins, we compare our model with the Fermi-LAT gamma-ray data and find the Poisson Likelihood, L [Mattox et al. (1996)]. For each DM channel and mass we then produce a Maximum Likelihood,

$$
\hat{L}_{j}\left(\theta_{D M}\right)=\prod_{i} P_{i j}\left(n_{i} ; \vec{\alpha}_{\max }, \theta_{D M}\right),
$$

by selecting the best-fitting linear parameters, $\vec{\alpha}$ (EGB, instrumental, and primary electron normalizations), for each DM normalization, $\theta_{D M}$. The $j$ represents the diffuse model and the product is over each bin, $i$.

Finally, we convert $\hat{L}$ into a Test Statistic for every GALPROP model that includes the CR- $\chi^{2}$ (Eq. 2.1) information,

$$
\lambda_{j}\left(\theta_{D M}\right)=\frac{P_{j}^{C R} \hat{L}_{j}\left(\theta_{D M}\right)}{\left(P_{j}^{C R} \hat{L}_{j}\right)_{b e s t}} .
$$




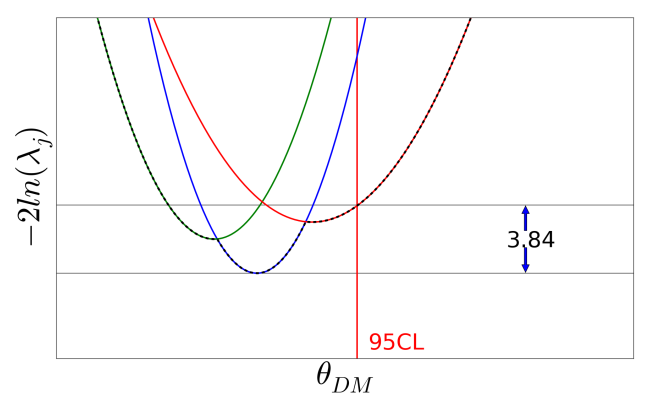

Figure 1: Qualitative plot of an upper limit set using the Profile Likelihood method on the Test Statistic defined in Eq. 3.2. Each curve represents a different GALPROP model.

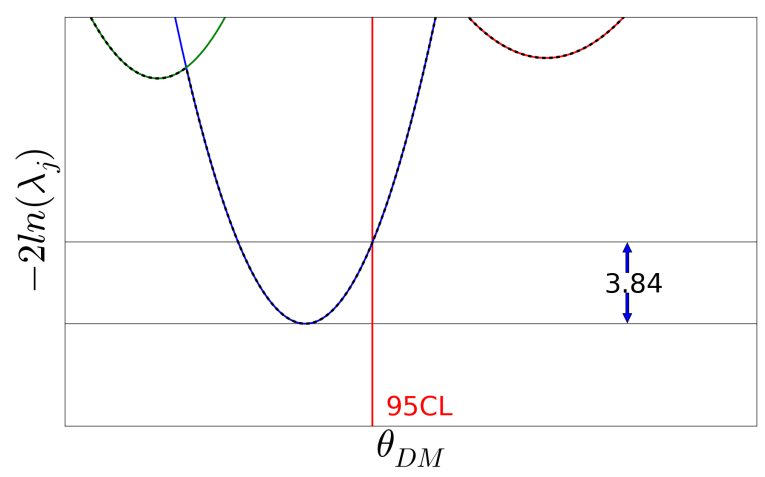

Figure 2: A qualitative plot of an under-sampled minimum in the Profile Likelihood.

This leaves us with a family of curves (Fig. 1), the minimum contour of which comprises the Profile Likelihood. The contour should behave as a $\chi^{2}$ with one degree of freedom, and we set our $95 \%$ confidence upper limit on $\theta_{D M}$ to where it rises above the minimum by 3.84 .

To be confident with the limit it is necessary to have sufficiently populated the model space such that the minimum is the true minimum, and that the nearby sampling is dense enough to smoothly detect the profile out to the upper limit. Under-sampling the parameter space leads to a situation like in Figure 2, where the limit is derived from a single model, and both the location of the true minimum and the shape of the curve around it are unclear.

After our initial, completely random, sampling of the parameter space in Table 1, we found ourselves in precisely that situation. Figure 3 illustrates the initial sampling as a scatter plot for the CR and gamma rays $\hat{L}$. The minimum will be near the lower left hand corner, and sampling should be densest around it. Clearly this was not the case.

\subsection{Classification Tree Sampling}

Being limited mainly by computation time, the best way to populate a specific region is to have a rough idea what its $\hat{L}$ will be beforehand. Predicting the gamma-ray fits would be problematic, due to the variety of DM signals being explored. The CR- $\chi^{2}$ is much more straight forward, and 


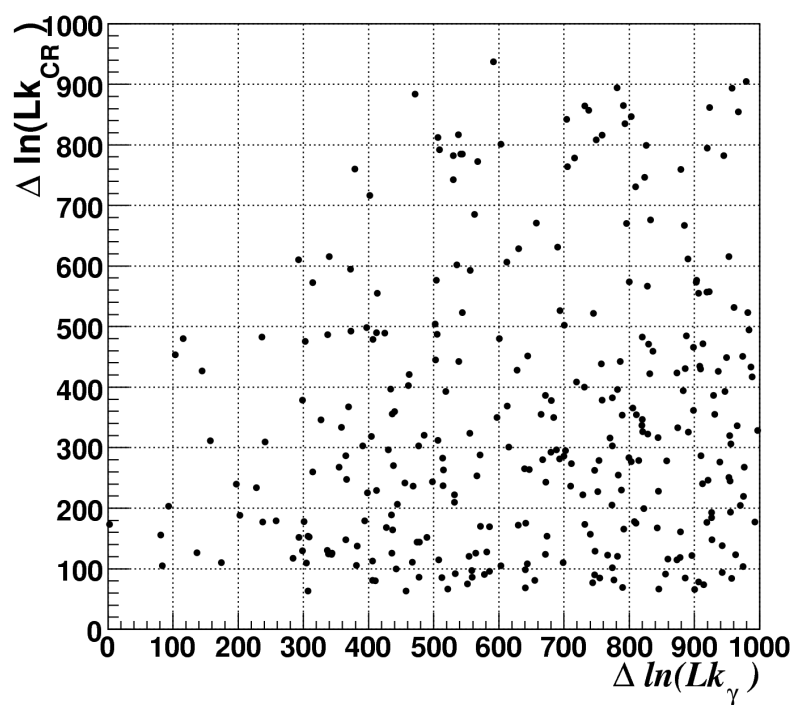

Figure 3: A scatter plot of the CR vs. gamma ray maximum Likelihood for our present models.

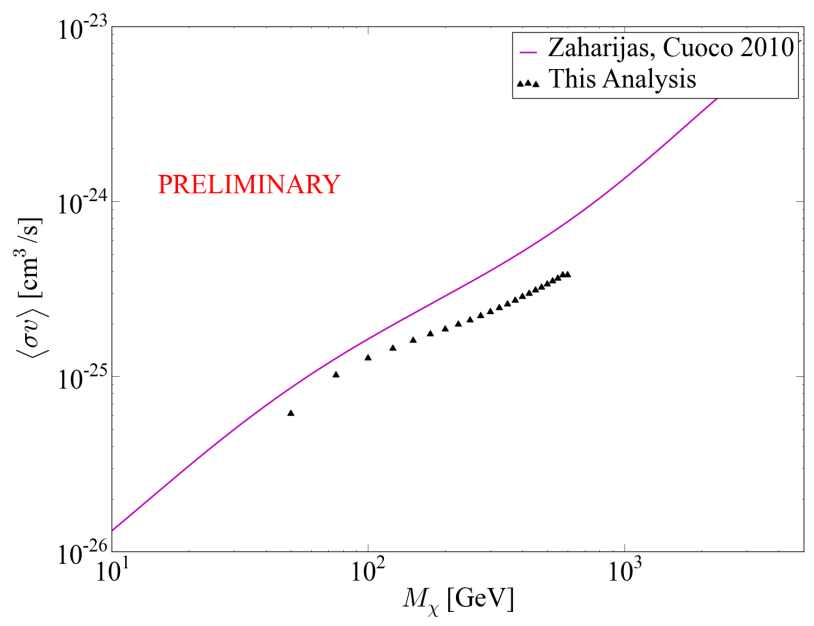

Figure 4: Present 95\% upper limits on pure $b \bar{b}$ annihilation from boosted Einasto DM. Also shown are $3 \sigma$ limits from an independent Fermi Collaboration analysis with an unboosted NFW profile, 4 kpc Halo height, and SNR-based CR source distribution [Zaharijas et al. (2010)] [Cuoco et al. (2010)].

so we use it to train a classification tree. The tree can then be used to pre-select GALPROP models that agree with CR data. This will reduce much of the y-axis scatter in Fig.3, hopefully allowing us to sufficiently sample the global minimum with our available computer resources.

\section{Preliminary Results}

Figure 4 shows our present $95 \%$ confidence upper limits on a pure $b \bar{b}$ channel WIMP. As 
mentioned earlier, these limits are based on a profile likelihood minimum composed of a single GALPROP model. This makes the limits very preliminary and it is unclear in which direction the final ones will move. Also shown in Fig. 4 are the closely-related limits derived by an independent Fermi Collaboration analysis which uses a single "conservative" GALPROP model with all of its gas (target) map contributions free to float.

A further caveat, there are persistent residuals even in our best fits. We hope to account for much of this by correcting known deficiencies to our model, foremost being the isotropic approximation to the inverse compton signal. We also plan on addressing uncertainty in the gas distributions by allowing some float in the collective contributions produced by interaction with molecular, atomic, and ionized Hydrogen. Given the level of uncertainties discussed above, these upper limits should be considered as purely illustrative of the Fermi LAT potential to constrain DM annihilation or decay from the Galactic halo.

\section{Summary and Outlook}

We have conducted a preliminary investigation of possible DM annihilation signal in the Milky Way Host Halo, and within the systematic uncertainties of our background model, find no significant detection. We model all known backgrounds, and use the Profile Likelihood method of marginalizing over the many nuisance parameters that come from uncertainty in the CR-induced galactic diffuse emission. Sampling the model parameter space adequately is a computationally difficult task, one we are addressing by intelligently pre-selecting our models with a classification tree. We expect the combination of increased sampling density of our maximum likelihood region and upcoming improvements to our models (anisotropic inverse compton \& inclusion of target uncertainty) to yield a robust limit that takes into account the large systematics inherent to a physical model of the galactic background.

\section{References}

[Abdo et al. (2010)] Abdo, A.A. et al. 2010, Phys. Rev. Lett. 104, 101101.

[Abdo et al. (2010)] Abdo, A.A. et al. 2010, APJS 188405.

[Cuoco et al. (2010)] Cuoco, A. et al. 2010, Identification of Dark Matter Conference.

[Diemand et al. (2007)] Diemand, J., Kuhlen, M., Madau, P. 2007, APJ 667, 859.

[Diemand et al. (2008)] Diemand, J., Kuhlen, M., Madau, P., Zemp, M., Moore, B., Potter, D., Stadel, J. 2008, Nature, 454, 735.

[Mattox et al. (1996)] Mattox, J. et al. 1996, APJ 461, 396.

[Strong et al. (2000)] Strong, A. W., Moskalenko, I. V., \& Reimer, O. 2004, APJ, 537, 763.

[Zaharijas et al. (2010)] Zaharijas, G. et al. 2010, Identification of Dark Matter Conference. 